the female to male ratio 5,5:1 in the north and 2:1 in the south. Time period from the first symptoms occurence to the RA diagnosis was 17.7 (13.2) months for the northern part and 25.0 (16.9) for the southern; 20.6 (14.9) for Serbia; in that period patients were mostly treated with NSAIDS (82\%) and physical therapy $(30 \%)$; short-lasting corticosteroids were given to $13 \%$, peroral corticosteroids to $4 \%$ and no patients were treated with DMARDS.

Conclusions: RA prevalence in the southern and northern part of Serbia is in line $(0.42 \%$ [95\% $\mathrm{Cl} 0.12 ; 0.72])$ vs $0.30 \%$ [ $95 \% \mathrm{Cl} 0.09 ; 0.51]$ ), being more frequently presented in females as compared to males (five times more in the north and two times more in the south). Delay in diagnosis as compared to the first symptoms occurence was 21 months and during that time no patients were treated with DMARDS.

References:

[1] Zlatkovic-Svenda MI, Stojanovic RM, Milenkovic MP, Vlajinac HD, Le Bihan E, Guillemin F. Adaptation and validation of a telephone questionnaire - Serbian version for case detection of rheumatoid arthritis and spondyloarthropathy (multicentric Eular study). Clin Exp Rheumatol. 2007;25 (1): 75-84.

Disclosure of Interest: None declared

DOI: 10.1136/annrheumdis-2017-eular.4899

\section{AB1150 SLEEP HEALTH AND QUALITY OF LIFE IN PATIENTS WITH KNEE OSTEOARTHRITIS BEFORE AND AFTER TOTAL KNEE REPLACEMENT}

M. Nuñez ${ }^{1}$, E. Nuñez ${ }^{2}$, J.M. Segur ${ }^{3}$, L. Lozano ${ }^{3}$, J. Montañana ${ }^{3}$, V. Segura ${ }^{3}$ M. Marti ${ }^{4}$, A. Garcia-Cardó ${ }^{4}$, S. Sastre ${ }^{3}$, X. Alemany ${ }^{3} .{ }^{1}$ Rheumatology and IDIBAPS Area 1, Hospital Clinic; ${ }^{2}$ SAP Suport al Diagnòstic i al Tractament, Institut Català de la Salut; ${ }^{3}$ Orthopedic Surgery and IDIBAPS; ${ }^{4}$ Rheumatology, Hospital Clinic, Barcelona, Spain

Background: Studies report that sleep disturbances are often associated with chronic musculoskeletal disease. There is no agreed definition of sleep health, but some characteristics, such as sleep duration (number of hours daily) and sleep quality or satisfaction (subjective evaluation of good or poor sleep) are used to evaluate sleep health. In a previous study in patients with severe osteoarthritis awaiting total knee replacement (TKR), patients reporting good quality sleep had better health-related quality of life (HRQL) measured by the specific WOMAC and generic SF-36 questionnaires.

Objectives: To measure sleep health in patients included on a waiting list for TKR and 12 months after TKR.

Methods: Prospective study with a 12-month follow up. Sociodemographic and clinical variables were determined. Sleep health: hours of sleep and reparative sleep (RS) were examined using the question "How well do you usually sleep?" measured on a Likert scale (1=good [RS], 2=regular, 3=badly, $4=$ with medication/treatment (non-reparative sleep [NRS]). Function and pain were measured using the WOMAC and SF-36 questionnaires. Comparisons were made using t-tests (paired samples) and McNemar's test. Linear regression models were used to analyze associations. Dependent variables: WOMAC and SF-36 pain and function dimensions; independent variables: sleep quality, age, sex, BMI, number of comorbidities, depression/anxiety.

Results: 105 patients ( $79 \%$ female, mean age 69.39 years [SD 8.3]) were included. $80 \%$ had $\geq 2$ comorbidities (mean 2.71 [SD 1.8]), mean BMI was 33.68 (SD 6.7), 32 had depression/anxiety, and mean sleep duration was 6.63 hours (SD 1.4). 12 months after TKF there were significant improvements in WOMAC dimension scores (mean $>25$ points, $p<0.001$ ) and SF-36 scores (mean $>19$ ). At study inclusion, $23 \%$ reported RS with a mean sleep duration of 7.5 hours (SD 1.1 ) vs. 6.24 hours (SD 1.5) in NRS patients ( $p=0.002) .12$ months after TKR, $40 \%$ of patients had RS $(p=0.029)$. Patients with RS had better scores in all quality of life dimensions $(<10$ points) than those with NRS $(p<0.05)$ at baseline and at 12 months. Multivariate analysis showed RS was independently associated with pain and function (WOMAC and SF-36) $(p<0.007)$.

Conclusions: Sleep health was associated with better HRQL before and after TKR. Although more patients had RS after TKR, $60 \%$ of patients continued not to have sleep health. Although often undervalued clinically, sleep health is closely associated with the health status.

Acknowledgements: This work was funded by project $\mathrm{Pl} / 13 / 00948$, integrated in the Plan Nacional I+D+I and cofounded by ISCIII-Subdirección General de Evaluación and European Regional Development Fund (ERDF).

References:

[1] Buysse DJ. Sleep health: can we define it? Does it matter? Sleep. 2014 Jan 1;37(1):9-17. doi: 10.5665/sleep.3298.

Disclosure of Interest: None declared

DOI: 10.1136/annrheumdis-2017-eular.1777

\section{AB1151 MONTH OF BIRTH AFFECTS THE RISK OF RHEUMATIC DISEASES: A NATIONWIDE CASE-CONTROL STUDY}

N. Koo, J. Lee, B.W. Lee, S.-K. Kwok, S.H. Park, J.H. Ju. Division of Rheumatology, Department of Internal Medicine, School of Medicine, The Catholic University of Korea, Seoul St. Mary's Hospital, Seoul, Korea, Republic Of

Background: There have been several studies which demonstrated the impact of birth on the risk of certain diseases such as asthma or cardiovascular diseases.
However, rheumatic diseases have not yet been thoroughly investigated in terms of association with birth month.

Objectives: In this study, we sought to determine whether birth month or season could affect the risk of rheumatologic diseases.

Methods: The birth month patterns of patients with rheumatic diseases were compared with to those of general population. We utilized the claims data of Health Insurance Review and Assessment Service (HIRA) which covers nearly $90 \%$ of total population in Korea. The associations between birth month/season and 32 diseases were investigated using logistic regression.

Results: Our dataset included 17,247,458 (male 8,224,670; female 9,022,788) individuals from HIRA database from January, 1997 to August, 2015. Among 27 rheumatic diseases, 8 diseases including Crohn's disease (CD), ulcerative colitis (UC), rheumatoid arthritis (RA), systemic lupus erythematosus, polymyalgia rheumatica (PMR), ankylosing spondylitis (AS), multiple sclerosis, gout, fibromyalgia (FMS) were significantly associated with birth month $(P<0.05)$. In terms of seasonality, CD, UC, RA, Sjogren's syndrome, PMR, AS, Gout, and FMS demonstrated significant difference. CD, UC and AS showed higher prevalence in individuals born in winter and lower prevalence in summer. On the other hand, people who were born in summer showed higher possibility to have gout and FMS compared to those born in winter. In consistent with previous reports, type 1 diabetes is more prevalent in those born in winter. Angina and myocardial infarction showed higher prevalence in patients born in spring and lower in fall. This consistency reflects the relevance of our dataset and methodology.

Conclusions: We found significant impacts of birth month/season on various rheumatic diseases. Seasonal variation of infective agents, sun exposure or food ingestion during gestation or early infancy may explain the association between birth month/season and certain disease development.

Disclosure of Interest: None declared

DOI: 10.1136/annrheumdis-2017-eular.2891

\section{AB1152 THE INCIDENCE OF HYPERSENSITIVITY TO NSAIDS IN THE GROUP OF PATIENTS WITH MUSCULOSKELETAL DISORDERS}

O. Brzezińska-Pawłowska ${ }^{1}$, A. Sztanka ${ }^{2}$, K. Słodkowski $^{2}$,

A. Lewandowska-Polak ${ }^{2}$, J. Makowska ${ }^{2}$. ${ }^{1}$ Department of Rheumatology, Immunology and Allergy; ${ }^{2}$ Department of Rheumatology, Medical Univercity of Lodz, Łódź, Poland

Background: Non-steroidal anti-inflammatory drugs (NSAIDs) are among the most common cause of drug hypersensitivity reactions (DHR). Recent studies show that the prevalence of hypersensitivity reaction to drug is particularly in Poland.

Objectives: To assess the frequency and the risk od developing NSAID hypersensitivity in patients with chronic disorders of the musculoskeletal system. Methods: The study group consisted of 200 patients (age 19-88 years - $54,5 \pm 14$, women-161, men-39) hospitalized in the Department of Rheumatology in 20152016. All patients filled questionnaire regarding symptoms of DHR after ingestion of non-steroidal anti-inflammatory drugs. The presence of DHR, clinical pattern of the reaction, frequency of NSAIDs administration and comorbidities have been studied. In statistical studies the main risk factors were defined.

Results: Seventy-seven patients from study group (38,5\%) reported symptoms that occurred within 24 hours after NSAIDs ingestion. Symptoms characteristic for hypersensitivity reaction were reported by 40 patients $(20 \%)$. Respiratory symptoms like dyspnea and/or cough were reported by 22 patients $(11 \%)$. Cutaneous symptoms (urticaria/angioedema/dermal flush) were reported also by 29 patients $(14,5 \%)$. Three patients experienced loss of consciousness. Thirty-seven patients reported isolated stomach cramps.

The symptoms developed usually between 2-12 hours after drug intake. In 37 patients oral administration caused DHR reaction, moreover in 14 patients also topical application led to adverse reaction. In most of patients reaction appeared due to COX-1 inhibitor and interestingly in 3 patients reaction was evoked by celecoxib which is perceived to be safe alternative for patients with NSAIDs hypersensitivity. Chronic urticaria, asthma and systematic drug intake occurs the main risk factors of hypersensitivity.

Conclusions: Drug hypersensitivity reactions are reported very frequently in population of patients with chronic musculoskeletal disorders. Our studies suggest that patients taking protractedly NSAIDs are in group with high risk of drug hypersensitivity development.

Disclosure of Interest: None declared

DOI: 10.1136/annrheumdis-2017-eular.5408

\section{AB1153 OSTEOPOROSIS RISK FACTORS IN PARTICIPANTS OF HEALTHY AGING ACADEMY}

O. Brzezińska-Pawłowska ${ }^{1}$, A. Łuczak ${ }^{2}$, D. Stalmach ${ }^{2}$, A. Lentas ${ }^{2}$,

M.L. Kowalski ${ }^{1}$, J. Makowska ${ }^{2} .{ }^{1}$ Department of Immunology, Rheumatology and Allergy; ${ }^{2}$ Department of Rheumatology, Medical Univercity of Lodz, Łódź, Poland

Background: Osteoporosis is a major cause of morbidity and mortality in elderly population. It is known that the mortality rate in European population within 1 year of hip fracture is about $30 \%$, and another $30 \%$ of patients need long term nursing for the rest of their life. However, the prevalence and associated risk factors in the Polish elderly population have not been well documented. The aim of the 
study was to assess prevalence of osteoporosis in Polish elderly population and to assess risk factors related to osteoporotic fractures

Objectives: The study was based on questionnaires distributed among 645 students ( 61 men, 533 women and 51 not report mean age 69,42 $\pm 5,88$ ) attending the Healthy Aging Academy at Medical University.

Methods: Students were asked to answer 20 questions including information about endured fractures, osteoporosis risk factors, screening densitometry, treatment of osteoporosis, supplementation with vitamin D3 and calcium. Also, questions about classical risk factors for osteoporosis (loss of height $(5 \mathrm{~cm} / \mathrm{year}$ ), family history, falls, loss of weight, treatment with steroids) were included to questionnaire.

Results: Fractures caused by fall from their own height were reported by 180 $(27,91 \%)$. Moreover, 96 (14,88\%) responders without fracture history declared more than $3 \mathrm{~cm}$ decrease of height after their forties, what strongly suggest osteoporotic vertebral fractures. Forty three (24\%) of the respondents had multiple fractures and single fractures were reported by $137(76 \%)$ respondents. The most common localization of fractures was forearm $(57 \%)$, shin bones $(10,4 \%)$ and foot bones (9\%). Among subjects who underwent densitometry after the fracture $32 \%$ had the diagnosis of osteopenia; $19,4 \%$ were diagnosed with osteoporosis and in $23,1 \%$ bone density was within normal limits. The strongest risk factors for osteoporotic fractures were numerous falls per year $(p=0,000)$ and frequent drinking of alcohol $(p=0,008)$. The risk factors of fractures (sum of risk factors for falls and osteoporosis) among people with previous fractures vs. those without fractures were statistically significant $(p=0,037)$. Only $13 \%$ of patient with fracture and $15 \%$ of all patients with osteoporosis and osteopenia were treated by antiosteoporotic drugs (ibandronian/aledronian). Calcium supplementation was received by $30 \%$ of patients with fracture and $43 \%$ supplemented vitamin D3.

Conclusions: The prevalence of osteoporotic fractures in population of relatively well educated population of participants of Third Age University seems to be high. Patients with osteoporosis do not receive appropriate pharmacological prophylactic treatment, pointing at the need for education of primary care physicians and elderly population about burden and consequences of osteoporosis.

Disclosure of Interest: None declared

DOI: 10.1136/annrheumdis-2017-eular.5452

\section{AB1154 CHARACTERIZATION OF CHILEAN PATIENTS WITH PULMONARY DISEASE ASSOCIATED TO A RHEUMATOLOGIC DIAGNOSIS IN AN INTEGRATED POLYCLINIC. HOSPITAL PADRE HURTADO EXPERIENCE}

O. Valenzuela ${ }^{1}$, V. Agar ${ }^{1}$, J.P. Leiva ${ }^{2}$, I. Allende ${ }^{2}$, G. Bozzano $^{2}$, G. Miranda ${ }^{3}$ S.E. Ibáñez Vodnizza ${ }^{1} .{ }^{1}$ Rheumatology department, Clinica Alemana de Santiago/Hospital Padre Hurtado; ${ }^{2}$ Facultad de Medicina Clinica Alemana de Santiago - Universidad del Desarrollo; ${ }^{3}$ Hospital Padre Hurtado, Santiago, RM, Chile

Background: Pulmonary diseases associated to a rheumatologic condition constitute a heterogeneous group of pathologies that are grouped according to their clinical and radiological similarity. There is a perception that the incidence of these diseases has been increasing, however, in Chile, there are no up-to-date publications regarding the characteristics of this group of patients.

Objectives: To describe and characterize patients with pulmonary disease associated to a rheumatologic diagnosis at Hospital Padre Hurtado, Chile, between the years 2014 and 2016.

Methods: Patients with suspected lung involvement associated to a connective tissue disease were evaluated by a bronchopulmonar specialist and a rheumatologist in a joint polyclinic. If the diagnosis was confirmed by the specialist the patient was included in the database. Data collected included age, gender, rheumatologic diagnosis and radiological pattern.

Results: 76 patients were included. $85.5 \%$ were female. The mean age was 58 years. Eighty-nine percent of the patients came from the Rheumatology department. These patients developed respiratory symptoms during the course

Table 1. Rheumatologic diagnosis of 76 patients

\begin{tabular}{lc}
\hline Rheumatoid Arthritis (\%) & $31(40.8)$ \\
Scleroderma (\%) & $8(10.5)$ \\
Inflammatory Myopathy (\%) & $5(6.6)$ \\
Sjögren Syndrome (\%) & $5(6.6)$ \\
Systemic Lupus Erythematosus (\%) & $4(5.3)$ \\
Undifferentiated Connective Tissue Disease (\%) & $3(3.9)$ \\
Mixed Connective Tissue Disease (\%) & $3(3.9)$ \\
Recurrent Polychondritis (\%) & $2(2.6)$ \\
Other diagnosis (\%) & $15(19.7)$ \\
\hline
\end{tabular}

Table 2. Radiological evaluation of 53 patients

Interstitial Lung Disease pattern: Non Usual Interstitial Pneumonia (\%) $26(49.1)$

Interstitial Lung Disease pattern: Usual Interstical Pneumonia (\%) $8(15.1)$

Bronchiolitis (\%)

Emphysema (\%)

Nodules (\%)

Pulmonary embolism (\%)

Bronchiectasis (\%)

Sarcoidosis (\%)

Tracheal stenosis (\%) of their disease. The remaining $11 \%$ were patients controlled in the Pulmonary diseases department, and the respiratory symptoms were the first manifestation of their conective tissue disease.The rheumatologic diagnoses that were concluded in this multidisciplinary polyclinic were distributed as described in Table 1.

$69.7 \%$ of the patients have completed the radiological evaluation (Table 2)

Conclusions: Having a multidisciplinary clinical unit allowed us to order and protocolize the study, follow-up and treatment of the patients with lung involvement associated with a connective tissue disease. Currently, the multidisciplinary management of these patients is indispensable, as described in the literature.

Disclosure of Interest: None declared

DOI: 10.1136/annrheumdis-2017-eular.4109

\section{AB1155 DEPRESSION AND SUICIDALITY ARE COMMON IN PSORIATIC ARTHRITIS AND AXIAL SPONDYLOARTHRITIS, AND RATES ARE COMPARABLE TO THOSE IN PSORIASIS}

A. Sheahan ${ }^{1}$, R. Suruki ${ }^{1}$, P.C. Taylor ${ }^{2}$, V.S. Sloan ${ }^{1} .{ }^{1}$ UCB Pharma, Raleigh, United States; ${ }^{2}$ Kennedy Institute, Oxford, United Kingdom

Background: Depression and suicidality are well-described comorbidities in psoriasis (PSO). The prevalence of these comorbidities in psoriatic arthritis (PsA) and axial spondyloarthritis (axSpA) is less well described.

Objectives: To assess the prevalence of depression and suicidality in PsA and axSpA in the recent literature, and compare rates to $\mathrm{PSO}$.

Methods: For PsA and axSpA, we evaluated the recent English-language literature identified through a PubMed search; we used a recent review and performed a targeted review of the period since the publication in order to establish the rates for PSO for comparison. ${ }^{1}$ Review articles were also examined to identify key publications.

Results: Rates of depression in PSO vary widely, depending on the outcome definition and method of ascertainment. Dowlatshahi et al. reported a pooled rate of $9.0-55.0 \%$, with rates from the literature after the review period ranging from $9.0-39.8 \%$. Rates for suicidality also varied widely, with $2.5-17.3 \%$ of patients (pts) reporting suicidal ideation. The limited data available provide ranges for depression in PsA of $3.4-28.6 \%$, and in axSpA of $3.1-44.0 \%$. The single study that differentiated between ankylosing spondylitis (AS) and non-radiographic (nr)-axSpA did not identify a difference between the two groups. ${ }^{2}$ Very limited data existed on suicidality in PsA and axSpA. For PsA, the incidence rates (IR) of suicidal ideation, attempts, and suicide per 1000 person-years in the UK were $0.4,1.3$, and $<0.001$, respectively ${ }^{3}$ no prevalence data were identified. In a study in China, $2.5 \%$ of pts with axSpA reported a past suicide attempt, ${ }^{4}$ while a study in Turkey found $9.6 \%$ of pts with AS had thoughts of suicide in the past year, but without plans. ${ }^{5}$ Depending on the study, different definitions and tools were used to assess depression (eg. HADS, ICD9, antidepressant use), and even when the same tool was used, different cutoffs for defining depression were implemented (eg. cutoff for HADS ranged from $\geq 5$ to $\geq 11$ ), making comparisons across studies difficult. In a large observational study of AS with matched controls, the IR of depression per 1000 pt-years was 5.48 in AS versus 3.29 without AS, risk ratio $1.63 .^{6}$

Conclusions: Although data are limited, rates of depression and suicidality in PsA and axSpA are comparable to those in PSO. Comparisons between studies and diseases are challenging due to a lack of standardized assessment tools and definitions of depression and suicidality. There are almost no data for nr-axSpA, which unlike AS has no gender predominance. Given that depression in PSO pts is more common in women, ${ }^{7}$ understanding the relative prevalence in AS versus $n r-a x S p A$ would be important. Generating additional data regarding the impact of depression and suicidality in PSA and axSpA should increase awareness among treating physicians.

References:

[1] Dowlatshahi EA. J. Invest Dermatol 2014;134:1542-51.

[2] Klic GE. Medicine (Baltimore) 2014:93:e337.

[3] Hagberg KW. Mod Rheumatol 2016;26:774-9.

[4] Chan CY. Int J Rheum Dis 2014; doi:10.1111/1756-185X.12456.

[5] Saygin C. Clin Exp Rheumatol 2015;33(6 Suppl 94):S30-5.

[6] Shen CC. J Rheumatol 2016;43:625-31.

[7] Lamb RC. Br J Dermatol 2016; doi:10.1111/bjd.14833

Disclosure of Interest: A. Sheahan Employee of: UCB Pharma, R. Suruki Employee of: UCB Pharma, P. Taylor: None declared, V. Sloan Employee of: UCB Pharma

DOI: 10.1136/annrheumdis-2017-eular.2455

\section{AB1156 THE DESCRIPTIVE EPIDEMIOLOGY OF CUTANEOUS LUPUS ERYTHEMATOSUS IN THREE LARGE U.S. ADMINISTRATIVE DATABASES, 2010-2014}

S.A. Hall ${ }^{1}$, L. Li ${ }^{2}$, S. Eaton ${ }^{2}$, C. Musselli ${ }^{2}$, A. Dilley ${ }^{1} .{ }^{1}$ Epidemiology; ${ }^{2}$ Biogen, Cambridge, United States

Background: Previous epidemiologic studies of cutaneous lupus erythematosus (CLE) have not described the pediatric burden, and have focused on specific geographic areas, limiting generalizability.

Objectives: To describe the demographics, comorbid conditions and pharmacy drug utilization of CLE patients, 3 large sources of U.S. administrative claims 